\title{
Trastorno mental y tipo de sustancia consumida: estudio piloto sobre la utilización de la Entrevista de Cribado de Patología Dual (ECDD)
}

\author{
Mario Sánchez-Morate Tristante', José Miñarro-López², Inmaculada Montoya-Castilla³, Marián Pérez-Marín \\ I Unidad de Conductas Adictivas de San Isidro, Consorcio Hospital General Universitario de Valencia. \\ 2 Departamento de Psicobiología. Unidad de Investigación en Psicobiología de las Drogodepedencias. Facultad de Psicología, Universidad de Valencia. \\ 3 Departamento de Personalidad, Evaluación y Tratamientos Psicológicos. Facultad de Psicología, Universidad de Valencia.
}

\section{RESUMEN}

Introducción: la Patología Dual se define como una enfermedad en la que concurren de forma simultánea en un mismo individuo, por lo menos, un trastorno por uso de sustancias y un trastorno mental. Ambos trastornos se influyen de manera mutua, por lo que se recomienda hacer un abordaje conjunto. Objetivo: analizar las características psicopatológicas y su asociación con determinados tipos de sustancias, a través de la Entrevista de Cribado en Diagnostico Dual (ECDD). Método: participaron 63 pacientes de entre 18 y 45 años, con una media de edad de 37.7 años ( $D T=9.6)$, asistidos en la Unidad de Conductas Adictivas de una población de Valencia. Las variables sociodemográficas, clínicas y toxicológicas se evaluaron a través del cuestionario del Sistema de Información para la Evaluación de la Calidad Asistencial en Drogodependencias (SECAD), en tanto que los diagnósticos de Patología Dual se analizaron a través de la Entrevista de Cribado en Diagnostico Dual (ECDD). Resultados: las sustancias que tuvieron mayores índices de demanda asistencial fueron la cocaína y el alcohol. En general, se encontró que $84.13 \%$ de los participantes presentaron algún tipo de trastorno del Eje I (uno o más) y que la sustancia que presenta mayor número de psicopatologías asociadas con su consumo es la cocaína. Discusión y conclusiones: la presencia de posibles casos de Patología Dual en la muestra evaluada con ECDD fue muy elevada; se observó una relación entre el tipo de sustancia consumida y la psicopatología diagnosticada. EI ECDD se presenta como una herramienta de gran utilidad en la práctica clínica diaria para la detección de posibles casos psicopatológicos en el campo de las adicciones.

Palabras clave: drogodependencias, trastornos por uso de sustancias, trastornos mentales, patología dual, instrumentos de orientación diagnóstica.

\begin{abstract}
Introduction: Dual pathology defines an illness case when a substance abuse disorder and a mental disorder simultaneously affect an individual patient. Both disorders influence each other, mutually, that its recommended what recommends to treat them together. Objective: to analyse the psychopatologic character and its association with different types of substances, evaluated through the Dual Pathology Screening interview (ECDD). Method: the participants were 63 patients between 18 and 45 years, with an average age of 37.7 years $(S D=9.6)$, assisted in the Unit of Addictive Behaviours of a Valencia town. The sociodemographic variables, clinical and toxycologic, were evaluated through the questionnaire from the Information System for the Evaluation of Quality Assistance in Drug Addiction (DRY). The Dual Pathology diagnostics were evaluated through the Dual Pathology Screening interview (ECDD). Results: substances with greater assistance demand were cocaine and alcohol. Our study indicates that $84.13 \%$ of the participants present some type of axis I disorder (one or more). Cocaine consumption presented the greater number of associated psychopathologies. Discussion: according to ECDD, the presence of possible cases of dual diagnosis in the sample was very high. Our study demonstrates a direct relationship between the substance type consumption and the psychopathological diagnosis. ECDD would appear to be a useful assessment tool in clinical practice for the detection of psychopathologies among patients with addictions.
\end{abstract}

Keywords: addictions, substance use disorders, mental disorders, dual pathology, diagnostic screening tools.

\footnotetext{
Autora de correspondencia:

Marián Pérez-Marín. Facultad de Psicología, Universidad de Valencia. Av. Blasco Ibáñez núm. 21, 46010, Valencia, España. Correo electrónico: marian.perez@uv.es

Fecha de recepción: 27 de julio de 2017

Fecha de aceptación: 12 de octubre de 2017

DOI: 10.28931/riiad.2017.2.03
} 


\section{INTRODUCCIÓN}

La Patología Dual (PD) se define como una enfermedad en la que concurren de forma simultánea en un mismo individuo, por lo menos, un trastorno por uso de sustancias (TUS) y un trastorno mental (TM; Torrens Mèlich, 2008; Szerman, 2009).

Los estudios señalan que los pacientes con PD -en comparación con quienes sólo tienen diagnóstico de drogodependencia o trastorno mental- presentan una mayor duración del cuadro clínico, tienen peor pronóstico, responden mal a los tratamientos y suelen mostrar una cantidad más elevada de recaídas (Baena Luna \& López-Delgado, 2006; Lieb, 2015); de la misma forma, acuden más a los servicios de urgencias (Martín-Santos et al., 2006), presentan más hospitalizaciones psiquiátricas (Rodríguez et al., 2008), mayores índices de policonsumo (Casares-López et al., 2011), una mayor prevalencia de comportamientos suicidas (Szerman et al., 2012), más conductas de riesgo que ponen en peligro su vida y una mayor incidencia de enfermedades infecciosas (Gilchrist, Blázquez, \& Torrens, 2011; Rosenberg et al., 2001). También presentan mayores tasas de desempleo y marginalidad (Barea et al., 2010), más problemas delictivos y legales originados por conductas violentas y criminales (Casares-López et al., 2011), y peores índices generales de calidad de vida, entendida como la percepción individual de su posición respecto a sus metas y expectativas, incluidas sus ideas sobre su salud física y psicológica (Astals et al., 2008; Benaiges Fuste, Prat i Vigué, \& Adán Puig, 2012).

La elevada prevalencia de esta comorbilidad supone dificultades tanto en el diagnóstico como en el tratamiento a corto, medio y largo plazo. Ambos trastornos se influyen de manera mutua y se recomienda hacer un abordaje conjunto, pues esto posibilita la realización de un mejor pronóstico (San et al., 2016). Se estima que cerca de la mitad de los drogodependientes actuales presentará una enfermedad mental en el próximo decenio; de igual manera, la mitad de los pacientes con enfermedades mentales abusarán de sustancias (Blanco, Sirvent, Moral, del Pilar Linares, \& Rivas, 2010).

Los estudios muestran una gran variabilidad respecto a la prevalencia de Patología Dual (Baena Luna \& López Delgado, 2006; European Monitoring Centre for Drugs and Drug Addiction [EMCDDA], 2015), misma que puede ser indicativa de la dificultad para realizar un diagnóstico correcto de la patología psiquiátrica en pacientes drogodependientes (Baena Luna \& López Delgado, 2006). Como señalan importantes estudios epidemiológicos, la PD es muy frecuente tanto en muestras de población general (Compton, Thomas, Stinson, \& Grant, 2007; Kessler, 2004; Kessler, Chiu, Demler, Merikangas,
\& Walters, 2005), como en muestras clínicas (Chan, Dennis, \& Funk, 2008); se han encontrado tasas de prevalencia que, dependiendo del estudio, oscilan entre 18 y 75\% (Rodríguez-Llera et al., 2006).

Por ello, en la actualidad es de especial interés utilizar instrumentos fiables y válidos para que los especialistas realicen un adecuado diagnóstico y un correcto abordaje terapéutico (Torrens, Mestre-Pintó, Montanari, Julian, \& Domingo-Salvany, 2017). Disponemos todavía, sin embargo, de escasos datos sobre la prevalencia de Patología Dual en población española y sobre la distribución de ésta en las dos redes principales de asistencia de estos problemas: la red de drogodependencias y la de salud mental (González-Saiz et al., 2011; Torrens, Gilchrist, \& Domingo-Salvany, 2011; Vázquez, 2010; Vergara-Moragues, et al., 2012).

Es importante que los servicios que atienden a este tipo de pacientes desarrollen una actitud diagnóstica activa con el propósito de identificar estos casos; de esta forma se conseguirá que el número de remisiones clínicas, el nivel de funcionamiento general y la calidad de vida de estas personas se vean incrementados (Torrens Mèlich, 2008).

En este sentido, el objetivo del presente estudio es analizar las características psicopatológicas y su asociación con determinados tipos de sustancias, a través de la Entrevista de Cribado en Diagnostico Dual (ECDD). Los objetivos específicos de este trabajo son: a) describir el perfil sociodemográfico y clínico de esta población; b) describir sus variables psicopatológicas; c) obtener perfiles psicopatológicos en función del tipo de sustancia o sustancias consumidas y la sintomatología clínica que presentan. Partimos de la hipótesis de que durante el estudio se observará una presencia elevada de $\mathrm{Pa}$ tología Dual y que el consumo de algunas sustancias estará asociado con más frecuencia a determinados trastornos psicopatológicos.

\section{MÉTODO}

\section{Criterios de inclusión}

Eran susceptibles de participar en el presente estudio todos los participantes que cumplían los siguientes requisitos: a) acudir, durante el tiempo que duró el estudio, a la Unidad de Conductas Adictivas (UCA) para solicitar asistencia terapéutica de desintoxicación o deshabituación por un problema de consumo de sustancias psicoactivas; b) ser mayores de edad; c) presentar abuso/ dependencia de sustancias psicoactivas; d) no tener alguna patología psiquiátrica diagnosticada previamente; e) reportar ausencia de tratamiento farmacológico (a excepción de los tratamientos sustitutivos por depen- 
dencia de opiáceos como metadona, buprenorfina y suboxone); f) aceptar voluntariamente la participación en la investigación.

Se excluyeron los participantes con adicciones comportamentales o sólo adicción a la nicotina.

Para la evaluación inicial del uso y las consecuencias del consumo de sustancias psicoactivas que presentaban los participantes del estudio, se utilizaron los criterios diagnósticos del DSM-IV-TR (American Psychiatric Association [APA], 2000).

\section{Participantes}

El presente trabajo se ha realizado en la UCA de una población de Valencia, perteneciente al Consorcio Hospital General Universitario de Valencia (CHGUV) de la Comunidad Valenciana, que atiende a una población de cerca de 121.000 habitantes.

Durante el tiempo que duró el estudio (marzo-junio de 2013), 79 participantes acudieron para solicitar una primera visita; se rechazaron a 16 por no cumplir con los criterios de inclusión. Los participantes incluidos fueron 63 pacientes de entre 18 y 45 años, residentes en valencia capital; la mayoría eran hombres (76.2\%), con una media de edad de 37.7 años $(D T=9.6) .38 \%(n=24)$ se situaba entre los 18 y 35 años $(M=28.09$; $D T=4.94)$; $40 \%(n=25)$ entre los 36 y $45(M=39.72 ; D T=2.44) ; y$ $22 \%(n=14)$ tenía más de 46 años $(M=50.62 ; D T=$ 3.33).

\section{Diseño}

Se utilizó un diseño descriptivo, relacional y transversal, con una técnica de muestreo consecutivo no aleatorio; se reclutó a los participantes que cumplían los criterios de inclusión, según acudían a la UCA. Los instrumentos de evaluación se administraron en un único momento temporal (en la primera visita concertada por el sujeto con la UCA, con una hora de duración estimada para el pase de instrumentos).

\section{Procedimiento}

El presente estudio fue aprobado para su realización por la Comisión de Investigación del Consorcio Hospital General Universitario de Valencia (CHGUV), tras formalizarse los documentos exigidos por el Comité Ético de Investigación Clínica (Documento de Confidencialidad de Datos, Modelo de Consentimiento Informado, Política de Protección de Datos y Documento Normalizado Declaración de Buena Práctica Clínica). A todos los participantes se les solicitó que firmaran el consentimiento informado para participar en el estudio.
Asimismo, se les realizó una toma de constantes (tensión arterial, frecuencia cardiaca, peso, talla e índice de masa corporal), una analítica de determinación de tóxicos en orina (barrido de sustancias: cannabis, cocaína, anfetamina, opiáceos, metadona y benzodiacepinas) y un control de alcoholemia, para determinar el consumo en el momento de iniciar el tratamiento y confirmar los datos obtenidos a través de la evaluación psicodiagnóstica.

\section{Variables e instrumentos de evaluación}

Las variables sociodemográficas, clínicas y toxicológicas se evaluaron a través del cuestionario Sistema de información para la Evaluación de la Calidad Asistencial en Drogodependencias (SECAD) y los criterios del DSMIV-TR de abuso y dependencia.

El cuestionario SECAD forma parte del protocolo utilizado en la Red Asistencial de Drogodependencias que valora la Asistencia Sanitaria como indicador de admisión al tratamiento y que se cumplimenta a través del programa informático ABUCASIS, proporcionado por la Conselleria de Sanitat de la Comunidad Valenciana.

Los diagnósticos de Patología Dual presentes en los participantes se evaluaron a través de la ECDD (Mestre, 2011), un instrumento de evaluación diseñado para la detección, en la práctica clínica, de los diagnósticos psicopatológicos comórbidos del Eje I del DSM más frecuentes entre los consumidores de sustancias psicoactivas. Consta de 66 preguntas subdivididas en seis apartados diagnósticos y uno de observaciones (Mestre, Domingo, Martín, \& Torrens, 2013). Los apartados diagnósticos son: a) estados de ansiedad (crisis de angustia/pánico, ansiedad generalizada, fobia simple, fobia social, agorafobia; b) estados de ánimo (distimia, depresión, manía); c) psicosis; d) Trastorno por Déficit de Atención e Hiperactividad (TDAH); e) Trastorno de Estrés Postraumático (TEPT). Cabe resaltar que con muestras españolas, la ECDD ha mostrado excelentes propiedades psicométricas (Mestre, 2011).

\section{Análisis estadístico de datos}

En el presente estudio se ha utilizado el programa SPSS en su versión Windows v.21.0. Se realizó un análisis de estadísticos descriptivos, en el que se calcularon frecuencias y porcentajes de las características sociodemográficas y las posibles patologías que aparecieran (a partir del SECAD y el ECDD).

Para determinar las probables relaciones estadísticamente significativas existentes entre las diferentes variables de estudio, se ha utilizado la prueba estadística no paramétrica de Chi-cuadrado de Pearson, con un 
intervalo de confianza de $95 \%$ de seguridad, dado que la mayoría de variables del estudio fueron nominales y cualitativas. Para comprobar las diferencias existentes entre las distintas categorías de las variables estudiadas se utilizó un análisis de residuales tipificados estandarizados.

\section{RESULTADOS}

\section{Perfil sociodemográfico y clínico de esta población}

Los pacientes mostraron un alto índice de enfermedades infectocontagiosas, como VIH (7.9\%) o hepatitis C (12.7\%). Dentro del área toxicológica, presentaron un alto índice de policonsumo: 88.9\% usaba, además de la droga principal, por lo menos otra sustancia psicoactiva.

El diagnóstico inicial, según los criterios de abuso y dependencia del DSM-IV-TR (APA, 2000), mostró que el diagnostico de dependencia (85.7\%) prevalecía frente al de abuso.

Las sustancias que tuvieron mayores índices de demanda asistencial fueron la cocaína (36.5\%) y el alcohol (28.5\%), seguidos a distancia por los opiáceos (16\%), la cannabis (14.2\%) y, para finalizar, las benzodiacepinas (4.8\%). Las mujeres reportaron más consumo de alcohol y benzodiacepinas, mientras que los hombres usaron más cocaína, opiáceos y cannabis. Los participantes mostraron un patrón de consumo prácticamente a diario, con una edad media de 18 años de inicio en el consumo de la sustancia por la que eran admitidos a tratamiento.

\section{Variables psicopatológicas}

Los resultados generales del estudio mostraron que $84.13 \%$ de los participantes presentaron algún tipo de trastorno del Eje I (uno o más).

Mediante el análisis, realizado con la ECDD, de las variables relacionadas con la aparición de posible psicopatología, se encontró que el consumo de sustancias está muy vinculado con los trastornos del estado de ánimo: aparecieron altos índices de sintomatología depresiva y de estrés postraumático (Tabla 1).

En el presente estudio, 51.9\% de los casos presentó psicopatología del estado de ánimo. Destaca, en especial, la sintomatología de tipo depresivo, con $82.5 \%$ del total de los casos, seguida por $55.6 \%$ de sintomatología maníaca y $17.5 \%$ de distímica. Los resultados de psicopatología psicótica muestran una presencia de 41.3\%; además, $31.70 \%$ de los pacientes presentó TDAH. Resalta la alta presencia de psicopatología de TEPT, con $55.6 \%$ de los casos.

Los resultados generales mostraron una presencia de $37.4 \%$ del conjunto de trastornos que componen la psicopatología ansiosa. Dentro de este apartado hay que destacar, en primer lugar, las crisis de pánico (58.6\%), seguidas de la ansiedad generalizada (46\%), la fobia social (44.4\%) y, por último, la fobia simple y la agorafobia (19\% cada una).

Al analizar los resultados globales en relación a las diferencias de sexo entre la población estudiada (Tabla 2), se observó una mayor presencia de prácticamente todos los trastornos en los hombres (pánico, ansiedad generalizada, fobia social, depresión, manía, psicosis, TDAH y TEPT), a excepción de la clínica agorafóbica y distímica, que estuvo más presente en las mujeres. La fobia simple apareció por igual en ambos sexos.

\section{Perfiles psicopatológicos en función del tipo de sustancia consumida y la sintomatología clínica (Patología Dual)}

A la hora de interpretar los resultados relacionados con la existencia de posible Patología Dual, hay que tener presente que el perfil de toxicomanía hace que se complique el diagnóstico, dado que la sintomatología puede ser causada por el policonsumo (88.9\%) o por la deprivación de las diferentes sustancias.

Al analizar los porcentajes de los cinco grupos de trastornos asociados con la sustancia consumida, se observó que (Figura 1): la droga que presenta mayor número de psicopatologías vinculadas con su consumo

Tabla 1

Presencia de trastornos psicopatológicos (evaluado con la escala ECDD)

\begin{tabular}{ccc}
\hline Trastorno psicopatológico & Presencia de trastorno & Ausencia de trastorno \\
$N(\%)$
\end{tabular}


Tabla 2

Presencia de psicopatología según sexo

\begin{tabular}{ccc}
\hline Trastorno psicopatológico & Mujer* & Hombre* \\
$N(\%)$ & $32(67.6)$ \\
\hline Estrés postraumático & $N(\%)$ & $38(80.0)$ \\
Déficit de atención e hiperactividad & $5(32.4)$ & $43(88.5)$ \\
Manía & $3(20.0)$ & $37(77.1)$ \\
Depresión & $2(11.5)$ & $36(75.0)$ \\
Distimia & $3(22.9)$ & $18(36.4)$ \\
Agorafobia & $4(25.0)$ & $20(41.7)$ \\
Fobia social & $10(63.6)$ & $36(75.0)$ \\
Fobia simple & $9(58.3)$ & $24(50.0)$ \\
Ansiedad generalizada & $4(25.0)$ & $33(69.0)$ \\
Pánico & $7(50.0)$ & $35(73.0)$
\end{tabular}

Nota: Total de mujeres:19; Total de hombres: 48.

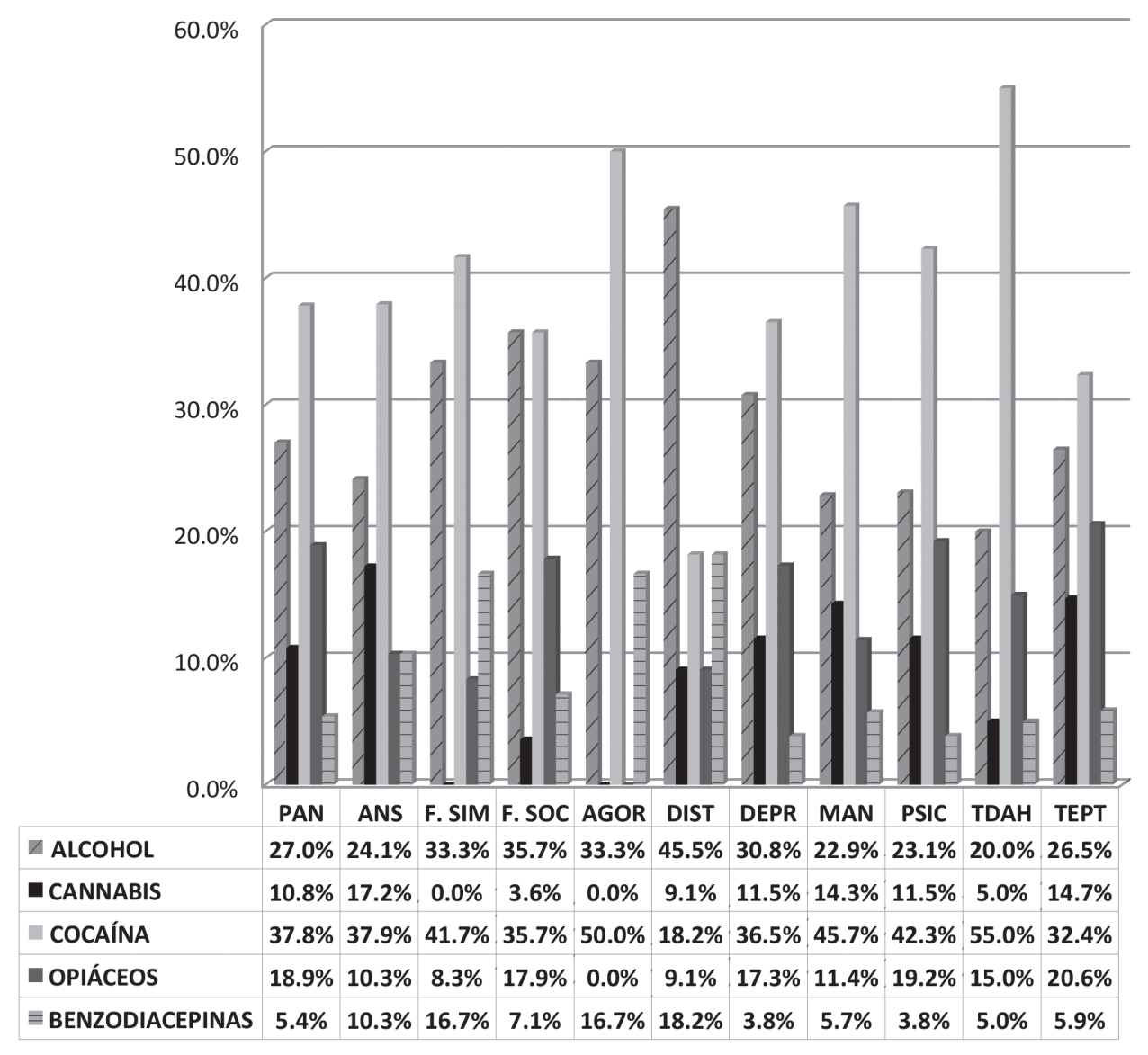

Figura 1. Psicopatologías según sustancia consumida. 
es la cocaína (40.7\%), seguida del alcohol (26.6\%) y los opiáceos (15.6\%); en cuarto lugar, se ubicaron las psicopatologías relacionadas con el consumo de cannabis, con 9.8\% de trastornos asociados; y, en último sitio, las benzodiacepinas, con $7.3 \%$ de psicopatología asociada.

\section{Análisis de la relación entre las variables de estudio}

Los resultados obtenidos sobre la relación entre variables muestran que según el sexo, los hombres presentan mayor índice de problemas judiciales $(41.7 \% \mathrm{H}$. vs $6.7 \%$ M.) $\left(X^{2}=6.300, p=.025\right)$; consumen mayoritariamente alcohol como segunda sustancia $(50 \% \mathrm{H}$. vs $13.3 \% \mathrm{M}$.) $\left(X^{2}=10.698, p=.031\right)$; y presentan mayor probabilidad de "no padecer" fobia simple $(82.4 \% \mathrm{H}$. vs $17.6 \% \mathrm{M}$.) $\left(x^{2}=5.605, p=.28\right)$. En contraste, las mujeres tienen mayor probabilidad de sufrir sintomatología agorafóbica $(58.3 \%$ M. vs $41.7 \%$ H. $)\left(X^{2}=9.740, p=.005\right)$ y distímica $\left(63.6 \%\right.$ M. vs $36.4 \%$ H.) $\left(X^{2}=11.653, p=.002\right)$.

Se encontraron diferencias significativas entre los grupos de edad: al comparar la sustancia consumida y los grupos de edad $\left(x^{2}=35.663, p=.000\right)$, se observó que los de 18 a 35 años consumieron cannabis como sustancia principal y con mayor frecuencia (55.6\%) que el resto de grupos de edad; los de entre 36 y 45 años usaron opiáceos (30\%); y los mayores de 46 , alcohol (casi $60 \%$ ); este último grupo de edad fue en el que más casos de fobia simple aparecieron.

Respecto de los trastornos psicopatológicos, sólo se encontraron diferencias estadísticamente significativas en el grupo de edad de mayores de 46 años, que reportó una mayor presencia de fobia simple $\left(X^{2}=9.808, p=.020\right)$; los participantes comprendidos en dicho grupo tenían una mayor probabilidad de padecer dicho trastorno, en concreto, 50\%. En el resto de los trastornos psicopatológicos no se encontró una relación significativa entre padecerlos y el rango de edad.

En relación con el consumo de sustancias, los opiáceos aparecieron relacionados con problemas judiciales $(70 \%)\left(X^{2}=12.343, p=.011\right)$ y la cocaína $\left(X^{2}=27.647\right.$, $p=.006)$, con una mayor frecuencia de consumo semanal $(17.4 \%)$ o de dos a tres días por semana (47.8\%). Las benzodiacepinas fueron las segundas sustancias más consumidas $(67.7 \%)\left(X^{2}=65.761, p=.001\right)$ y las que más se relacionaron con la psicopatología, de hecho, $6.7 \%$ de los casos $\left(x^{2}=9.739, p=.043\right)$ tenía síntomas de agorafobia. No aparecieron otras relaciones significativas entre sustancias consumidas y psicopatología.

\section{DISCUSIÓN Y CONCLUSIONES}

Los resultados obtenidos aportan evidencias en la línea de lo planteado en la hipótesis de investigación, en cuanto a que se observaría una presencia elevada de Patología Dual y que el consumo de algunas sustancias estaría asociado con más frecuencia a determinados trastornos psicopatológicos.

En el presente estudio se observó un alto índice de enfermedades infectocontagiosas asociadas con el consumo de sustancias psicoactivas, tal como lo reportaron estudios previos (Arias et al., 2013; Gilchrist et al., 2011; Rosenberg et al., 2001). Asimismo, se encontró un alto índice de policonsumo, también observado en los trabajos consultados, con una alta prevalencia que oscila entre 70 y 90\% (Casares-López et al., 2011; Domínguez-Martin, Miranda-Estribí, Pedrero-Pérez, Pérez-López, \& Puerta-García, 2008; Pérez-Abad, Rodríguez-Quirós, Ochoa Mangado, Ripoll Sevillano, \& Arranz Peña, 2006).

Mediante la evaluación realizada con la ECDD, podemos señalar que la población de consumidores que acudieron a la UCA para solicitar asistencia terapéutica manifestó una alta proporción conjunta de trastornos por uso de sustancias y psicopatología; destacaron, de mayor a menor presencia: los TEPT, los trastornos del estado de ánimo, los psicóticos, los ansiosos y el TDAH.

Los resultados globales dentro del área psicopatológica mostraron una elevada presencia $(84.1 \%)$ de participantes con posibles trastornos psicopatológicos. Existen diferentes estudios donde se encuentran también altas prevalencias de Patología Dual, con cifras que, dependiendo del estudio, oscilan entre 30 y 80\% (Álvarez González, 2009; Gual, 2007; Rodríguez-Llera et al., 2006).

La mayoría de los pacientes, alrededor de $80 \%$, presentó sintomatología de tipo depresivo. Otros estudios muestran resultados similares respecto de los síntomas afectivos (Miquel, Alonso, Ortiz, \& Brugué, 2011; Szerman, Basurte, Roncero, Martínez-Raga, \& Vega, 2008; Torrens \& Martínez-Sanvisens, 2009).

Se encontró, además, una presencia de psicopatología psicótica de $41.3 \%$. Algunos estudios han reportado cifras de prevalencia de trastornos psicóticos similares, pero existe una gran variabilidad de los datos y las cifras que relacionan el consumo de sustancias con los trastornos psicóticos llegan hasta 70-80\% (Rodríguez et al., 2008; Szerman et al., 2011; Westermeyer, 2006).

Se identificó, asimismo, que $31.7 \%$ de los pacientes presentaba un trastorno por déficit de atención e hiperactividad. Los estudios señalan una fuerte interrelación del trastorno por déficit de atención e hiperactividad con el de uso de sustancias, entre 15 y 50\% (Cumyn, French, \& Hechtman, 2009; Ochoa, Salvador, \& Madoz-Gúrpide, 2010; Torrico Linares et al., 2012).

La psicopatología del TEPT se observó en $55.6 \%$ de los casos. Los estudios revisados muestran índices de prevalencia elevados de esta psicopatología, pero por debajo de los encontrados en este estudio, con cifras 
de $46 \%$ (Miquel et al., 2011) o 51.8\% a lo largo de la vida (Reynolds et al., 2005). Entre las posibles razones de las cifras superiores encontradas en nuestro estudio, podría estar la utilización de una herramienta que podría ser más sensible a detectar el trastorno.

Se ha observado que $37.4 \%$ de los pacientes presentan psicopatología ansiosa. Diversos estudios han encontrado prevalencias generales de los trastornos de ansiedad similares, apareciendo gran variabilidad en los resultados (13-60\%) dependiendo del tipo de trastorno y de la sustancia consumida (Baena Luna \& López Delgado, 2006; Barea et al., 2010; Szerman et al., 2011; Vergara-Moragues et al., 2012).

Las conclusiones generales extraídas al analizar las posibles psicopatologías mostraron que: a) el TDAH, la agorafobia y la manía son los principales trastornos psicopatológicos relacionados con el consumo de cocaína; b) la distímia y la fobia social y simple están fundamentalmente vinculados con el consumo de alcohol; c) el trastorno de estrés postraumático, los trastornos psicóticos y las crisis de pánico se relacionan sobre todo con el consumo de opiáceos; d) la ansiedad generalizada, el trastorno de estrés postraumático y la manía se relacionan, sobre todo, con el consumo de cannabis; e) la distímia, la agorafobia y las crisis de pánico se asocian con el consumo de benzodiacepinas.

Una posible explicación de algunos de estos resultados podría estar en la hipótesis de la automedicación: los participantes buscarían, en las diferentes sustancias consumidas, paliar los síntomas de los cuadros que presentan. En el tratamiento del TDAH se utilizan los psicoestimulantes y en las crisis de ansiedad, los tranquilizantes o ansiolíticos; así, los participantes con estas psicopatologías buscarían sustancias que los alivien de la sintomatología que presentan; por ejemplo, los participantes con TDAH usarían cocaína y los ansiosos, cannabis.

A su vez, el uso repetitivo de sustancias podría generar, en participantes con predisposición, cuadros psicopatológicos por acción directa o indirecta de la sustancia consumida. Por ambas razones, de una forma o de otra, la evaluación, el diagnóstico y el tratamiento se complican en estos participantes con Patología Dual.

Los perfiles que se pueden extraer de los resultados obtenidos muestran que la población estudiada corresponde a una población adulta, en la que la media de edad de solicitud de demanda asistencial se sitúa en torno a los 37 años; los hombres, en general, consumen más cocaína, opiáceos y cannabis, y reportan una mayor presencia de Patología Dual relacionada con prácticamente todos los trastornos analizados (crisis de pánico, ansiedad generalizada, fobia social, trastornos psicóticos, TDAH y TEPT), a excepción de los síntomas agorafóbicos y distímicos. Por su parte, las mujeres consumen más alcohol y benzodiacepinas y muestran una mayor presencia de Patología Dual relacionada con la agorafobia y la distímia. Estos datos son similares a los emitidos por el informe 2012 del EMCDDA. En la fobia simple, ambos sexos mostraron presencias similares.

Estos resultados fueron sorprendentes dado que la mayoría de estudios (Chen, et al., 2011; Gorbea \& de Madrid, 2012; Miquel et al, 2011; Ochoa \& Madoz-Gúrpide, 2008) reportan mayores porcentajes de trastornos ansiosos, del estado de ánimo y TEPT entre las mujeres que entre los hombres; pero sí coinciden con los encontrados en los hombres para los trastornos psicóticos y TDAH, con mayores porcentajes entre éstos en comparación con las mujeres.

En cuanto a las limitaciones, se debe mencionar que al ser este un estudio piloto con una muestra reducida, los resultados se consideran preliminares, por lo que son necesarios más acercamientos que utilicen este instrumento de evaluación en una muestra mayor. En cuanto a la fortaleza principal, la investigación utilizó una herramienta ágil en la práctica diaria de las unidades de conductas adictivas.

En definitiva, nuestros resultados muestran una elevada presencia de patología dual en la población de consumidores de sustancias psicoactivas. La entrevista de cribado en diagnóstico se presenta como una herramienta útil y práctica en la clínica diaria, para detectar la posible presencia de Patología Dual, lo que puede facilitar el trabajo del personal que atiende a este tipo de pacientes.

\section{AGRADECIMIENTOS}

Agradecemos a los pacientes participantes en este estudio; al Consorcio Hospital General Universitario de Valencia por su autorización para la realización de la investigación; a los compañeros de la UCA de San Isidro, por su apoyo y colaboración; y a la Fundación Instituto Mar de Investigaciones Médicas, por el trato recibido durante el proceso de formación en la entrevista ECDD.

\section{FUENTES DE FINANCIACIÓN}

Este artículo ha sido realizado gracias a la financiación recibida por el Ministerio de Economía y Competitividad, el Instituto de Salud Carlos III y la Red de Trastornos Adictivos (RTA) RD12/0028/0005.

\section{CONFLICTOS DE INTERESES}

No existen conflictos de intereses. 


\section{REFERENCIAS}

Álvarez González, F. J. (2009). Información sobre la evaluación de patología dual en pacientes drogodependientes de Castilla y León. Revista Española de Drogodependencias, 34(2), 228-235.

American Psychiatric Association. (2000). Diagnostic and Statistical Manual of Mental Disorders (4ta. ed.). Washington, D.C.: Autor.

Arias, F., Szerman, N., Vega, P., Mesias, B., Basurte, I., Morant, C., ... Babin, F. (2013). Abuso o dependencia a la cocaína y otros trastornos psiquiátricos. Estudio Madrid sobre la prevalencia de la patología dual. Revista de Psiquiatría y Salud Mental, 6(3), 121-128. doi: 10.1016/j.rpsm.2012.09.002

Astals, M., Domingo-Salvany, A., Buenaventura, C. C., Tato, J., Vázquez, J. M., Martin-Santos, R., ... Torrens, M. (2008). Impact of substance dependence and dual diagnosis on the quality of life of heroin users seeking treatment. Substance Use \& Misuse, 43(5), 612-632. doi:10.1080/10826080701204813

Baena Luna, M. R., \& López Delgado, J. (2006). Trastornos duales. Mecanismos etiopatogénicos. Trastornos adictivos, 8(3), 176181. doi:10.1016/s1575-0973(06)75118-2

Barea, J., Delegido, A. B., López, M. R., Hernández, C. M., Martín, E., López, N., ... Cortés, G. H. (2010). Estudio sobre aspectos etiológicos de la patología dual. Adicciones: Revista de Socidrogalcohol, 22(1), 15-24. doi:10.20882/adicciones.210

Benaiges Fuste, I., Prat i Vigué, G., \& Adán Puig, A. (2012). Health-related quality of life in patients with dual diagnosis: clinical correlates. Health and Quality of Life Outcomes, 10(1), 106. doi:10.1186/1477-7525-10-106

Blanco, P., Sirvent, C., Moral, M. V., del Pilar Linares, M., \& Rivas, C. (2010). Psicopatología y género en adicciones. $11^{\circ}$ Congreso Virtual de Psiquiatría. España: Interpsiquis. Portal Virtual de Psiquiatría.

Casares-López, M. J., González-Menéndez, A., Bobes-Bascarán, M. T., Secades, R., Martínez-Cordero, A., \& Bobes, J. (2011). Necesidad de evaluación de la patología dual en contexto penitenciario. Adicciones: Revista de Socidrogalcohol, 23(1), 37-44. doi:10.20882/adicciones. 165

Chan, Y. F., Dennis, M. L., \& Funk, R. R. (2008). Prevalence and comorbidity of major internalizing and externalizing problems among adolescents and adults presenting to substance abuse treatment. Journal of Substance Abuse Treatment, 34(1), 14-24. doi:10.1016/j.jsat.2006.12.031

Chen, K. W., Banducci, A. N., Guller, L., Macatee, R. J., Lavelle, A., Daughters, S. B., ... Lejuez, C. W. (2011). An examination of psychiatric comorbidities as a function of gender and substance type within an inpatient substance use treatment program. Drug and Alcohol Dependence, 118(2-3), 92-99. doi:10.1016/j. drugalcdep.2011.03.003

Compton, W. M., Thomas, Y. F., Stinson, F. S., \& Grant, B. F. (2007). Prevalence, correlates, disability and comorbidity of DSM-IV drug abuse and dependence in the United States: results from the national epidemiologic survey on alcohol and related conditions. Archives of General Psychiatry, 64(5), 566. doi: 10.1001/archpsyc.64.5.566
Cumyn, L., French, L., \& Hechtman, L. (2009). Comorbidity in adults with attention-deficit hyperactivity disorder. The Canadian Journal of Psychiatry, 54(10), 673-683. doi:10.1177/070674370905401004

Domínguez-Martín, A. L., Miranda-Estribí, M. D., Pedrero-Pérez, E. J., Pérez-López, M., \& Puerta-García, C. (2008). Estudio de las causas de abandono del tratamiento en un centro de atención a drogodependientes. Trastornos Adictivos, 10(2), 112-120. doi: 10.1016/s1575-0973(08)74553-7

European Monitoring Centre for Drugs \& Drug Addiction. (2012). Annual report on the state of the drugs problem in the European Union. Lisboa: Office for Official Publications of the European Communities.

European Monitoring Centre for Drugs \& Drug Addiction. (2015). Annual report on the state of the drugs problem in the European Union. Lisboa: Office for Official Publications of the European Communities. Recuperado de http://www.emcdda.europa.eu/ edr2015

Gilchrist, G., Blázquez, A., \& Torrens, M. (2011). Psychiatric, behavioural and social risk factors for HIV infection among female drug users. AIDS and Behavior, 15(8), 1834-1843. doi:10.1007/ s10461-011-9991-1

González-Saiz, F., Lozano, O. M., Martín, J., Bilbao, I., Ballesta, R., \& Gutiérrez, J. (2011). Comorbilidad psiquiátrica en una muestra de pacientes con dependencia de opiáceos tratados con buprenorfina sublingual en régimen de comunidad terapéutica. Revista de Psiquiatría y Salud Mental, 4(2), 81-87. doi:10.1016/j. rpsm.2011.01.003

Gorbea, C. P. \& de Madrid, C. (2012). Psychiatric disorders associated with alcoholism: 2 year follow-up of treatment. Actas Españolas de Psiquiatría, 40(3), 129-35.

Gual A. (2007). Dual diagnosis in Spain. Drug and Alcohol Review, 26(1), 65-71. doi:10.1080/09595230601037000

Kessler, R. C. (2004). The epidemiology of dual diagnosis. Biological Psychiatry, 56(10), 730-737. doi: doi:10.1016/j.biopsych.2004. 06.034

Kessler, R. C., Chiu, W. T., Demler, O., \& Walters, E. E. (2005). Prevalence, severity, and comorbidity of 12-month DSM-IV disorders in the National Comorbidity Survey Replication. Archives of General Psychiatry, 62(6), 617-627. doi:10.1001/archpsyc.62.6.617

Lieb, R. (2015). Epidemiological perspectives on comorbidity between substance use disorders and other mental disorders. En G. Dom, \& F. Moggi (Eds.). Co-occurring Addictive and Psychiatric Disorders (pp. 3-12). Berlin, Heidelberg: Springer Berlin Heidelberg. doi:10.1007/978- 3-642-45375-5_1

Martín-Santos, R., Fonseca, F., Domingo-Salvany, A., Ginés, J. M., Imaz, M. L., Navinés, R., ... Torrens, M. (2006). Dual diagnosis in the psychiatric emergency room in Spain. The European Journal of Psychiatry, 20(3), 147-156. doi:10.4321/s021361632006000300002

Mestre, J. I. (2011). Evaluación de una entrevista de cribado para la detección de comorbilidad psiquiátrica en sujetos consumidores de sustancias de abuso (Tesis doctoral). Barcelona: Universidad Autónoma de Barcelona. 
Mestre, J. I., Domingo, A., Martín, R., \& Torrens, M. (2013). Dual diagnosis screening interview to identify psychiatric comorbidity in substance users: development and validation of a brief instrument. European Addiction Research, 20(1), 41-48. doi: 10.1159/000351519

Miquel, L., Alonso, C. R., Ortiz, C. L., \& Brugué, M. C. (2011). Diferencias de género epidemiológicas y diagnósticas según Eje I en pacientes con Patología Dual. Adicciones: Revista de Socidrogalcohol, 23(2), 165-172. doi: 10.20882/adicciones. 160

Ochoa, E., \& Madoz-Gúrpide, A. (2008). Diferencias de género en el tratamiento de la dependencia de opiáceos con naltrexona. Actas Españolas de Psiquiatría, 36, 197-204.

Ochoa, E., Salvador, E., \& Madoz-Gúrpide, A. (2010). Trastorno por déficit de atención e hiperactividad (TDAH) en adolescentes consumidores de sustancias. $11^{\circ}$ Congreso Virtual de Psiquiatría. España: Interpsiquis. Portal Virtual de Psiquiatría.

Pérez-Abad, M., Rodríguez-Quirós, J., Ochoa Mangado, E., Ripoll Sevillano, E., \& Arranz Peña, M. I. (2006). Monitorización analítica de drogas de abuso en el medio hospitalario. Trastornos Adictivos, 8(4), 236-242. doi: 10.1016/s1575-0973(06)75129-7

Reynolds, M., Mezey, G., Chapman, M., Wheeler, M., Drummond, C., \& Baldacchino, A. (2005). Co-morbid post-traumatic stress disorder in a substance misusing clinical population. Drug and Alcohol Dependence, 77(3), 251-258. doi: 10.1016/j.drugalcdep. 2004.08.017

Rodríguez-Llera, M. C., Domingo-Salvany, A., Brugal, M. T., Silva, T. C., Sanchez-Niubo, A., \& Torrens, M. (2006). Psychiatric comorbidity in young heroin users. Drug and Alcohol Dependence, 84(1), 48-55. doi: 10.1016/j.drugalcdep.2005.11.025

Rodríguez, R., Aragüés, M., Jiménez, M., Ponce, G., Muñoz, A., Bagney, A., ... Hoenicka, J. (2008). Patología dual en pacientes psiquiátricos hospitalizados: prevalencia y características generales. Investigación Clínica, 49(2), 195-205.

Rosenberg, S. D., Goodman, L. A., Osher, F. C., Swartz, M. S., Essock, S. M., Butterfield, M. I., ... Salyers, M. P. (2001). Prevalence of HIV, hepatitis $B$, and hepatitis $C$ in people with severe mental illness. American Journal of Public Health, 91(1), 31-37. doi: 10.2105/ajph.91.1.31

San, L., Arranz, B., Arrojo, M., Becoña, E., Bernardo, M., Caballero, L., ... Zorrilla, I. (2016). Clinical guideline for the treatment of dual pathology in the adult population. Adicciones, 28(1), 3-5. doi: 10.20882/adicciones.784

Szerman, N. (2009). Patología Dual. Protocolos de intervención: introducción. Barcelona: Edikamed S.L.
Szerman, N., Arias, F., Vega, P., Babín, F., Mesias, B., Basurte, I., ... Poyo, F. (2011). Estudio piloto sobre la prevalencia de patología dual en pacientes en tratamiento en la Comunidad de Madrid. Adicciones, 23(3), 249-256. doi: 10.20882/adicciones. 149

Szerman, N., Basurte, I., Roncero, C., Martínez-Raga, J., \& Vega, P. (2008). Patología dual: conductas adictivas y otros trastornos mentales. Jano: Medicina y Humanidades, 1(1720), 57-60.

Szerman, N., López-Castroman, L., Arias, F., Morant, C., Babín, F., Mesías, B., ... Baca-García, E. (2012). Dual diagnosis and suicide risk in a Spanish outpatient sample. Substance Use and Misuse, 47(4), 383-389. doi: 10.3109/10826084.2011.636135

Torrens, Mèlich (2008). Patología dual: situación actual y retos de futuro. Adicciones: Revista de socidrogalcohol, 20(4), 315319. doi: $10.20882 /$ adicciones. 255

Torrens, M., Gilchrist, G., \& Domingo-Salvany, A. (2011) Psychiatric comorbidity in illicit drug users: substance-induced versus independent disorders. Drug and Alcohol Dependence, 113(2-3), 147-156. doi: 10.1016/j.drugalcdep.2010.07.013

Torrens, M., \& Martínez-Sanvisens, D. (2009). Patología Dual. Protocolos de intervención: depresión. Barcelona: Edikamed S.L.

Torrens, M., Mestre-Pintó, J., Montanari, L., Julian, V., \& Domingo-Salvany, A. (2017). Patología Dual: una perspectiva europea. Adicciones, 29(1), 3-5. doi: 10.20882/adicciones.933

Torrico Linares, E., Vélez Moreno, A., Villalba Ruiz, E., Fernández Calderón, F., Hernández Cordero, A., \& Ramírez López, J. (2012). TDAH en pacientes con adicción a sustancias: análisis de la prevalencia y de los problemas relacionados con el consumo en una muestra atendida en un servicio de tratamiento ambulatorio. Trastornos Adictivos, 14(3), 89-95. doi: 10.1016/ s1575-0973(12)70050-8

Vázquez, F. L. (2010), Psychoactive substance use and dependence among Spanish university students: prevalence, correlates, polyconsumption and comorbidity with depression. Psychological Reports, 106(1), 297-313. doi: 10.2466/pr0.106.1.297-313

Vergara-Moragues, E., González-Saiz, F., Lozano, O. M., Betanzos, P., Fernández, F., Bilbao-Acebos, I., ... Verdejo, A. (2012) Psychiatric comorbidity in cocaine users treated in therapeutic community: substance-induced versus independent disorders. Psychiatry Research, 200(2-3), 734-741. doi: 10.1016/j.psychres. 2012.07.043

Westermeyer, J. (2006). Comorbid schizophrenia and substance abuse: a review of epidemiology and course. American Journal of Addictions, 15(5), 345-355. doi: 10.1080/10550490600860114 